\title{
Fast and Discriminative Semantic Embedding
}

\author{
Rob Koopman \\ OCLC Research \\ rob.koopman@oclc.org
}

\author{
Shenghui Wang \\ OCLC Research \\ shenghui.wang@oclc.org
}

\author{
Gwenn Englebienne \\ University of Twente \\ g.englebiennedutwente.nl
}

\begin{abstract}
The embedding of words and documents in compact, semantically meaningful vector spaces is a crucial part of modern information systems. Deep Learning models are powerful but their hyperparameter selection is often complex and they are expensive to train, and while pre-trained models are available, embeddings trained on general corpora are not necessarily well-suited to domain specific tasks. We propose a novel embedding method which extends random projection by weighting and projecting raw term embeddings orthogonally to an average language vector, thus improving the discriminating power of resulting term embeddings, and build more meaningful document embeddings by assigning appropriate weights to individual terms. We describe how updating the term embeddings online as we process the training data results in an extremely efficient method, in terms of both computational and memory requirements. Our experiments show highly competitive results with various state-of-the-art embedding methods on different tasks, including the standard STS benchmark and a subject prediction task, at a fraction of the computational cost.
\end{abstract}

\section{Introduction}

Modern information systems rely extensively on the embedding of words and documents in compact, semantically meaningful vector spaces, where semantic similarity/relatedness can be computed and used efficiently. Various embedding methods are essentially all based on the Distributional Hypothesis (Harris, 1954, Sahlgren, 2008), and rely on co-occurrence evidence found in a corpus - whether computed globally or in a local context.

The recent success of local context predictive models such as Word2Vec (Mikolov et al., 2013) have initiated the development of more complex and powerful deep learning models (Bojanowski et al., 2016, Peters et al., 2018). The resulting embeddings combine compactness and discriminating ability, but the associated computational requirements are substantial and the optimal hyperparameter settings are not easy to find. It is, therefore, more common that embeddings are pre-trained on large corpora and plugged into a variety of downstream tasks (sentiment analysis, classification, translation, etc.). However, such transfer learning might fail to capture crucial domain-specific semantics.

Revisiting the methods based on global co-occurrence counts, high dimensional spaces built from the raw global co-occurrence counts are normally mapped to a more compact, lower-dimensional space of embeddings, using dimensionality reduction methods such as Principal Component Analysis (Pearson, 1901), Locally Linear Embeddings (Roweis and Saul, 2000), and Random Projection (Achlioptas, 2003, Johnson and Lindenstrauss, 1984). The latter has the unique advantage of being computationally cheap in the creation of the low-dimensional space, while being a linear projection method for which no optimisation is required at test time. On the flip side, the lack of optimisation means separation of datapoints is obtained by a comparatively larger increase of the dimensionality, and the linearity of the method further limits how compact the low-dimensional representation can be. However, thanks to the simplicity of the model, very efficient optimisations can be made to the algorithms and the resulting embeddings can be made to be effective even in high-dimensional spaces. 
In our approach, we use a two-step process where we first reduce the dimensionality of the term vectors by an extremely efficient implementation of random projection. We then project the term vectors on the hyperplane orthogonal to the average language vector, which improves how discriminative the vector representations are, and assign appropriate weights for building document embeddings.

The main contributions of this papers are:

- a method that computes the Random Projection of terms as expressed in terms of their co-occurrences in documents without storing the matrix of term co-occurrences,

- a method to compensate for uninformative language elements by projection on the hyperplane orthogonal to the average language vector,

- an effective weighing, adaptable to the domain and empirically verified, of the resulting vectors which optimises the discriminative power of terms, and

- on the whole, a practical method which requires no iterative parameter optimisation and computes embeddings and associated weights in a single pass through the documents of the training corpus.

As we show in our experiment section, the resulting method is highly competitive with the state-ofthe-art, in terms of sentence similarity computation and downstream classification task, and has much lower computational space and time requirements.

\section{Related work}

Term embedding Much research has adopted the notion of Statistical Semantics (Furnas et al., 1983, Weaver, 1955) or in Linguistics the Distributional Hypothesis (Harris, 1954, Sahlgren, 2008). Various distributional semantic models have been proposed to represent (embed) words in a continuous vector space where semantically similar words are mapped to nearby points ('are embedded nearby each other'). The approaches fall into two main categories (Baroni et al., 2014). First, methods based on global co-occurrence counts (e.g., Latent Semantic Analysis (Dumais, 2005)) which compute statistics of how often some word co-occurs with its neighbour words in a large text corpus, and then use dimensionality-reduction methods (e.g., Singular-Value Decomposition (Trefethen and Bau III, 1997), Random Projection (Achlioptas, 2003; Johnson and Lindenstrauss, 1984, QasemiZadeh et al., 2017)) to map the co-occurrence statistics of each word to a small, dense vector. Second, local context predictive methods (e.g. neural probabilistic language models) which directly try to predict a word from its neighbours or vice versa in terms of learned small, dense embedding vectors. The recent successes in the latter models, e.g. Word2Vec (Mikolov et al., 2013; Baroni et al., 2014) have initiated the development of more complex models with deep learning, such as FastText (Bojanowski et al., 2016), ElMO (Peters et al. 2018). However, that also brings high computational cost and complex parameters to optimise.

Document embedding There are currently many competing deep learning schemes for learning sentence/document embeddings, such as Doc2Vec (Le and Mikolov, 2014), lda2vec (Moody, 2016), FastText (Bojanowski et al. 2016), Sent2Vec (Pagliardini et al., 2018), InferSent (Conneau et al., 2017), etc. These are generally powerful, but are comparatively computationally very expensive. A simple baseline such as averaged word embeddings is fast and still gives strong results in the annual Semantic Textual Similarity task, as reported by Cer et al. (2017). However, assigning the proper weights to words when calculating the sentence/document embedding is non-trivial. Arora et al. (2017) proposed to remove a common component (remove the projection of the vectors on the first principal component of the test set's representation) after sentences are embedded as the weighted average of word embeddings. This is a relatively cheap but effective improvement, but 1) it requires re-adapting the term vectors to each new set of sentences, 2) it requires performing Singular-Value Decomposition, which is $O\left(n^{3}\right)$ and therefore still quite challenging in the case of large datasets, and 3) the subtraction of the first principal component does not reduce the rank of the model: it improves the discriminative power in practice, but does not combat over-fitting. 
We propose a model that addresses these three issues: it is only trained on the training set; it does not require any optimisation (its computational time is linear in the size of the training set and can be implemented extremely efficiently); it uses linear projection to reduce the rank of the model. With only two straightforward parameters to tune - the term vectors' dimensionality and the minimum threshold below which rare words are discarded — the simplicity of our model is also a desirable feature in practice.

\section{Algorithm}

Let a document be a set of words for which co-occurance is relevant $\left.\right|^{1}$ and $n_{D}$ be the total number of documents and $n_{V}$ the number of frequent terms ${ }^{2}$ A term is frequent when it occurs in more than $K$ documents in the corpus, where $K$ is flexible depending on the size of the corpus. As we show in Section 5.1.2, however, with the weightings we propose in our approach, $K$ can be very low. $D$ is the chosen dimensionality of the embedding vectors.

Further, let $v_{a}$ be the $D$-dimensional average vector of the training documents. For each frequent term $t \in V$, we have the following parameters: $c_{t}(d)$, The number of occurrences of term $t$ in document $d ; d_{t}$ the number of documents that term $t$ occurs in; $\vec{v}_{t}$ the raw embedding vector of term $t ; \vec{r}_{t}$ a $D$ dimensional "random vector" for term $t$, i.e., $\vec{r}_{t}$ is a row of $\mathbf{R}$ (In our approach, this vector is binary and contains an equal number of +1 and -1 ); $w_{t}$ the weight assigned to term $t$ for document embedding.

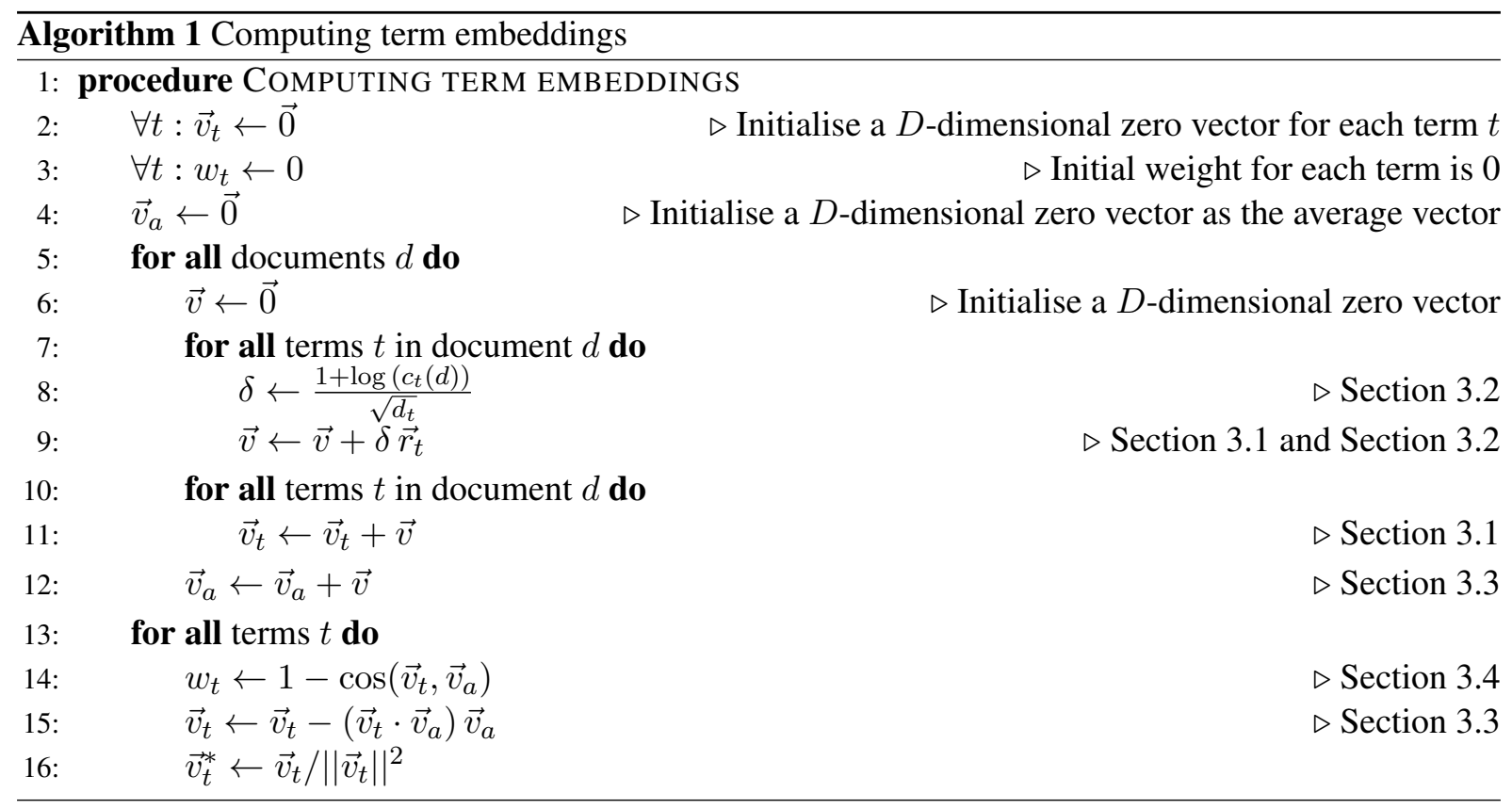

\subsection{Fast Random Projection}

Traditional random projection starts by computing a matrix of (weighted) term co-occurrences $\mathbf{C}$ of size $n_{V} \times n_{V}$, where $n_{V}$ is the total number of terms. This matrix contains, for each pair of terms $t_{i}$ and $t_{j}$, the number of documents (or paragraphs, or sentences) of the corpus in which both $t_{i}$ and $t_{j}$ occur. Using a matrix of random projection vectors $\mathbf{R}$ of size $n_{V} \times D$, we can then project our $n_{V}$-dimensional representation of each term to a lower $D$-dimensional space:

$$
\mathbf{C}_{\left[n_{V} \times D\right]}^{\prime}=\mathbf{C}_{\left[n_{V} \times n_{V}\right]} \mathbf{R}_{\left[n_{V} \times D\right]}
$$

\footnotetext{
${ }^{1}$ In this context, a document could therefore be a sentence, a paragraph, a fixed-size window, a bibliographic record, etc.

${ }^{2}$ Terms could be words or phrases. Common phrases are automatically detected using a method similar to that described by Mikolov et al. (2013).
} 
However, computing $\mathbf{C}^{\prime}$ requires us to store both $\mathbf{C}$ and $\mathbf{R}$, which can be challenging in terms of storage space and unacceptably expensive for large vocabularies. Instead, we propose a method to compute $\mathbf{C}^{\prime}$ without ever explicitly representing $\mathbf{C}$, simply by leveraging the linear nature of the projection (Eq. 1), updating $\mathbf{C}^{\prime}$ directly as we go through the corpus.

Algorithm 1 does this by decomposing $\mathbf{C}$ in Eq. 1 into the sum of individual documents' cooccurrence matrices, $\mathbf{C}=\sum_{d} \mathbf{C}_{d}$, so that

$$
\mathbf{C}^{\prime}=\sum_{d} \mathbf{C}_{d} \mathbf{R}
$$

where $\mathbf{C}_{d}$ is a matrix of zeros and ones indicating whether two terms co-occurred in the document. Importantly, we can further decompose the matrix multiplication by relying on the properties of $\mathbf{C}_{d}$ : every term in the document co-occurs equally with every other term in the document, so that all of the document's terms contribute equally to the projection. We can, therefore, sum all the relevant rows of $\mathbf{R}$ and add the result to the relevant rows of $\mathbf{C}^{\prime}$, making the time complexity linear in the size of the corpus, and the space complexity linear in the vocabulary size and constant in the number of documents .

\subsection{Weighted counts}

To improve the robustness of the approach, we weight the co-occurrence matrix $\mathbf{C}$ to reduce the effect of terms that are extremely common in certain documents and of terms that occur in the vast majority of documents. We use the term's average modified TF.IDF score in the training documents. Experimentally, we verified that the traditional IDF term of $\log \frac{N}{d_{t}}$ suppresses frequent terms too much, and replace it by a factor of $\sqrt{N / d_{t}}$, which has a similar effect but a longer tail and can also be seen as the normalisation constant of the t-test statistic (Manning and Schütze, 1999). For the TF term, we use a factor of $1+$ $\log c_{t}(d)$ and ignore the constant $N$ which cancels out in the subsequent normalisation. Each row of $\mathbf{R}$ is therefore weighted accordingly in the decomposition outlined above.

\subsection{Orthogonal projection}

Traditional models discard both very infrequent words (because they are too rare for the model to be able to capture their semantics from the training data) and very frequent words (so-called "stop words" because they do not provide any semantically useful information). In our approach, we give a continuous weight to terms based on how frequently they occur and compute the average "language vector" of the corpus, $\vec{v}_{a}$. Unsurprisingly, this vector is very similar to the average vector of stop words. Intuitively, words are increasingly more informative as they differ more from the average vector. By this reasoning, we project word vectors on the orthogonal hyperplane to $\vec{v}_{a}$ (Algorithm 1 line 15) ${ }_{3}^{3}$ resulting in a representation where the uninformative component of terms is eliminated, and normalise the vectors to have unit length. When computing document vectors, we down-weight terms according to their similarity to $\vec{v}_{a}$ (see Section 3.4). This step is crucial to get distinctive document embeddings.

As a nice side effect, projection makes it possible to handle multilingual corpora. The vocabulary of one language tends to be largely orthogonal to that of other languages (since words of one language tend to co-occur almost exclusively with words of the same language), so that projection using one language's average vector does not have much effect on the terms in other languages. This makes it possible to handle different languages effectively, within the same vector space.

\subsection{Term weight assignment}

Using the projection described above, the component that differentiates a term from the average vector is kept as its final embedding. Similarly, how different a term is from $\vec{v}_{a}$ also indicates how much that term contributes to the semantics of a document it is part of. In fact, we can interpret the cosine similarity as

\footnotetext{
${ }^{3}$ We use projection rather than subtracting $\vec{v}_{a}$ to prevent orthogonal vectors from gaining undue importance.
} 
a lower bound on the mutual information (MI) between the two vectors (Foster and Grassberger, 2011). In order to give a higher weight to the most informative terms, we assign a higher weight to words with lower MI by setting the final weight of each term to be $w_{t}=1-\cos \left(\vec{v}_{t}, \vec{v}_{a}\right)$.

\subsection{Text embedding}

With the frequent terms' embedding vectors and their proper weights, we can compute text embedding as the weighted average of its component term embeddings. For a text $\mathcal{T}$, we obtain a set of vectors $\mathcal{V}=\left\{\vec{v}_{t_{1}}^{*}, \ldots, \vec{v}_{t_{n}}^{*}\right\}$, where $n$ is the number of terms in text $\mathcal{T}$ and $\vec{v}_{t_{i}}^{*}$ is the final embedding vector for term $t_{i}$. The embedding of text $\mathcal{T}$ is calculated as follows:

$$
\vec{v}_{d}=\frac{\sum_{i=1}^{n} w_{t_{i}} \cdot \vec{v}_{t_{i}}^{*}}{\sum_{i=1}^{n} w_{t_{i}}}
$$

where $w_{t_{i}}$ is the weight for term $t_{i}$ and out-of-vocabulary words are ignored. Note how term and document vectors all have unit length, making similarity computations elegant and effective.

\section{Experimental methodology}

We performed three experimental evaluations: in Experiment 1, we compare our method to the state-ofthe-art on the standard STS benchmark; in Experiment 2, we qualitatively evaluate the weights assigned to terms; in Experiment 3, we evaluate our embedding method in terms of subject prediction. For our experiments, we implemented a parallelised version of the algorithm in C. All experiments were carried out on the same server with 2 Intel Xeon Silver 4109T 8-core processors and 384GB memory.

For meaningful evaluation, we trained all methods on the same two datasets using publicly available code for the state-of-the-art methods, and compared the resulting models on the standard STS benchmark. One dataset is the generic Simple English Wikipedia. ${ }^{4}$ The other domain-specific one is a subset of the MEDLINE database that consists of $10^{6}$ MEDLINE articles, randomly selected from WorldCat . org. In the latter dataset, each article is written in English and has a title and an abstract, to ensure sufficient textual information for computing the word embeddings. This dataset is of interest to our research and provides an interesting use case, as it consists of scientific articles and contains an above-average proportion of technical terms and jargon. Very rare terms carry critical meaning and make the task of word embedding particularly challenging.

\section{Experimental results}

\subsection{Experiment I: STS Benchmark and Computational efficiency}

The Semantic Textual Similarity (STS) Benchmark $5^{5}$ is a SemEval task organized between 2012 and 2017. It consists of 8628 pairs of English sentences, selected from image captions, news headlines and user forums. The similarity between these sentence pairs was annotated using a five point scale via crowdsourcing (Agirre et al. 2016). Participating systems calculate the similarity between these sentence pairs and are evaluated based on their Pearson correlation with the gold standard STS annotations.

We trained our method on the September 2018 datadump of Simple English Wikipedia, where we applied a sliding window of 80 terms, with $50 \%$ overlap. This resulted in 628,382 windows, each being considered as a separate document for co-occurrence counting. A total of 199,430 unique 256dimensional term vectors were obtained, their weights were calculated (Section 3.4), and used to embed the sentences in the STS benchmark (Section 3.5). The results are listed in Table 1], together with several state-of-the-art methods on the STS benchmark as published by Cer et al. (2017).

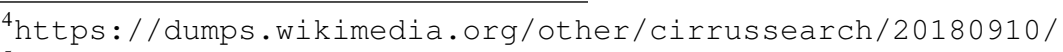

shttp://ixa2.si.ehu.es/stswiki/index.php/STSbenchmark 
Table 1: STS scores and train times of different methods and different settings.

\begin{tabular}{lllll}
\hline Method & Dev & Test & Dataset used & Train time \\
\hline Doc2Vec (PV-DBOW) & 72.2 & 64.9 & AP-NEWS & Unknown \\
& 31.0 & 27.0 & S.E. Wikipedia & $23 \mathrm{~m}$ \\
& 53.7 & 49.8 & MEDLINE & $2 \mathrm{~h} 3 \mathrm{~m}$ \\
FastText & 65.3 & 53.6 & Wikipedia & Unknown \\
& 48.6 & 38.9 & S.E. Wikipedia & $51 \mathrm{~m}$ \\
& 52.8 & 41.1 & MEDLINE & $3 \mathrm{~h} 4 \mathrm{~m}$ \\
Sent2Vec & 78.7 & 75.5 & Twitter & Unknown \\
& 68.8 & 62.2 & S.E. Wikipedia & $18 \mathrm{~m}$ \\
& 65.4 & 55.0 & MEDLINE & $3 \mathrm{~h} 10 \mathrm{~m}$ \\
Our method & 75.1 & 64.6 & S.E. Wikipedia & $17 \mathrm{~s}$ \\
& 73.6 & 58.9 & MEDLINE & $43 \mathrm{~s}$ \\
\hline Effect of projection and weighting in our method & \\
\hline No projection, no weighting & 31.4 & 34.2 & MEDLINE & $43 \mathrm{~s}$ \\
No projection, TFIDF weighting & 45.3 & 46.3 & MEDLINE & $43 \mathrm{~s}$ \\
With projection, no weighting & 57.3 & 45.2 & MEDLINE & $43 \mathrm{~s}$ \\
With projection, TFIDF weighting & 68.4 & 55.8 & MEDLINE & $43 \mathrm{~s}$ \\
With projection, our weighting & 73.6 & 58.9 & MEDLINE & $43 \mathrm{~s}$ \\
\hline
\end{tabular}

We then ran the publicly available implementations of Word2Vec (Mikolov et al. 2013), Doc2Vec (Le and Mikolov, 2014), GloVe (Pennington et al., 2014), FastText (Bojanowski et al., 2016) and Sent2Vec (Pagliardini et al., 2018) and trained them on the two datasets using the same machine. The common hyperparameters were chosen to generally maximise the different methods' performance for this task, and are: a vector size of 256 , a minimal number of word occurrences of 10 , number of negative samples of 10, window size of 10, using hierarchical softmax, a learning rate of 1.0 and a number of threads of 16. All the other parameters were kept to their default values. When trained on the MEDLINE dataset, for word embedding only, it took Word2Vec and GloVe 29 and 35 minutes, respectively. As sentencelevel embedding methods, Doc2 Vec cost more than 2 hours to train. FastText and Sent2Vec also required more than 3 hours. In contrast, our combined term and document embedding - which includes the $10^{6}$ Medline articles and $340 \times 10^{3}$ unique terms - requires only $43 \mathrm{~s}$.

In analysing the STS benchmark results (Table 1), it is apparent that our method substantially outperforms all baseline methods when trained on the same dataset. Also notice how the training dataset has a clear impact on each method's performance, and even though the Simple English Wikipedia dataset is more limited, both in vocabulary and in size, than the datasets used for publication by the other methods, our method still outperforms the published results of the other baselines in terms of STS scores, and is very competitive with Sent2Vec. All methods suffer a drop of performance on the generic STS benchmark when trained on the MEDLINE dataset, as a consequence of the domain-specific nature of the dataset, but this drop is least pronounced in the case of our method. This suggests that a careful search for a more appropriate training set would improve the method's performance even further.

Finally, we should emphasise how low the train times are for our method. Since we do not require any iterative optimisation of the model parameters, our method's results are deterministically determined by the training data, they do not depend on parameter initialisation, and training is orders of magnitudes faster than the other methods.

\subsubsection{Effect of projection and weighting}

We here report the effects of projection on the hyperplane orthogonal to $\vec{v}_{a}$ (see Section 3.3) and weighting (see Section 3.4 in our method. As shown in Table 1, the projection greatly improves our method 
Table 2: STS scores and train times w.r.t. $K$

\begin{tabular}{rrrcr}
\hline$K$ & Dev & Test & Train time $(\mathrm{s})$ & \#terms \\
\hline 5 & 73.9 & 60.5 & $55 \mathrm{~s}$ & 753,422 \\
10 & 73.6 & 58.9 & $43 \mathrm{~s}$ & 339,729 \\
20 & 71.5 & 57.8 & $37 \mathrm{~s}$ & 183,058 \\
40 & 69.0 & 55.2 & $35 \mathrm{~s}$ & 109,662 \\
80 & 65.7 & 52.1 & $32 \mathrm{~s}$ & 68,461 \\
\hline
\end{tabular}

in terms of STS scores. Our weighting also substantially outperforms TFIDF weighting (both with and without projection), which itself outperforms no weighting. The difference in train time is negligible.

\subsubsection{Effect of $K$}

Decreasing the threshold $K$ below which terms are ignored, results in a disproportionate increase of the number of terms that are included, with the computed vectors for those added terms being increasingly noisy. Because of the added computational burden and the noisiness of the estimation, traditionally a comparatively large cutoff value for $K$ is chosen. With our proposed method, however, very small values for $K$ are practical and the runtime does not grow much when a smaller $K$ is chosen.

Table 2 reports the effect of $K$ on the STS benchmark and the corresponding runtime, when training with the $10^{6}$ MEDLINE articles. With a $K$ as small as 5, the runtime stays reasonable, while it brings real benefits in terms of the STS benchmark, beyond those reported in Table 11(where all methods use $K=10$ ), because more infrequent terms get embedded.

\subsection{Experiment II: Qualitative evaluation of weights for individual terms}

Table 3 gives examples of terms with their raw document counts and final weights. As expected, traditional stop words such as "for" and "also" have extremely low weights. Frequent terms such as "treatment," "analysis," "system," and "subsequent" are to some extent domain-specific stop words which have low semantic value and therefore low weights too. However, more meaningful or discriminative terms such as "inflammatory," "mRNA," "antibodies" and "immune" have much higher weights even when they are also used very frequently.

At the other end of the spectrum, less frequent terms are likely to carry discriminative information for representing the semantics of the whole documents; however not all equally infrequent words have equally high weights. For example, "comprised" and "clarify" have much lower weights than "cytometry," "spleen," "cox" and "embryos" which are expected to be key topics for documents which contain them. The orthogonal projection and weighting help to give discriminative terms a boost when calculating the document embedding, no matter how frequently these terms are used.

In addition, we observed that the average cosine similarity between all documents is smaller by orders of magnitude when orthogonal projection and weighting is performed compared to when it is not, suggesting the documents are distributed in more compact clusters. That being said, without a proper evaluation with domain experts, it is not easy to evaluate the genuine validity of such operation. Our future work will include conducting such user-in-the-loop evaluation.

\subsection{Experiment III: Subject prediction}

In most digital library catalogs, bibliographic records are indexed using controlled vocabularies or thesauri to improve the discoverability of the content. These vocabularies are either generic, such as Library of Congress Subject Headings (LCSH) 6 or domain-specific, such as Medical Subject Headings (MeSH) which is used for indexing articles in the MEDLINE database. Traditionally, assigning a most relevant

thttp://id.loc.gov/authorities/subjects.html

https://www.nlm.nih.gov/mesh/ 
Table 3: Examples of term counts and their adjusted weights

\begin{tabular}{lrc}
\hline$t$ & $d_{t}$ & $w_{t}$ \\
\hline for & 720,776 & 0.003322 \\
also & 230,896 & 0.024318 \\
treatment & 171,984 & 0.079871 \\
analysis & 170,669 & 0.042365 \\
system & 99,582 & 0.077036 \\
inflammatory & 27,743 & 0.356823 \\
mRNA & 27,681 & 0.318550 \\
achieved & 27,382 & 0.114748 \\
antibodies & 27,379 & 0.433778 \\
subsequent & 27,289 & 0.060512 \\
\hline
\end{tabular}

\begin{tabular}{lcc}
\hline$t$ & $d_{t}$ & $w_{t}$ \\
\hline comprised & 6099 & 0.293460 \\
timing & 6098 & 0.336269 \\
artificial & 6093 & 0.465012 \\
cytometry & 6086 & 0.776044 \\
adjuvant & 6085 & 0.501349 \\
spleen & 6080 & 0.523253 \\
mucosal & 6080 & 0.505713 \\
cox & 6072 & 0.608585 \\
embryos & 6055 & 0.724599 \\
clarify & 6053 & 0.298637 \\
\hline
\end{tabular}

subset of subject headings to describe a record is done manually by professional taxonomists. However, such manual assignment is very time-consuming and can no longer keep up with the speed at which new records are produced. Therefore automatically assigning a set of relevant subjects to articles becomes increasingly important.

We evaluated our embedding method on the use case of subject prediction. This remains a difficult problem and is a form of Extreme Multi-label Text Classification (XMTC) (Prabhu and Varma, 2014, Bhatia et al., 2015, Liu et al., 2017), where the prediction space normally consists of hundreds of thousands to millions of labels and data sparsity and scalability are the major challenges. In our MEDLINE dataset, there are more than 324,619 MeSH headings indexing 896,300 articles (the other articles do not have any subjects) with on average 16 headings per article. However, only 102,484 MeSH headings are used to index more than 10 articles.

We propose to treat the MeSH headings as terms in the documents they are associated with, so that terms, documents and MeSH headings are all embedded in the same $D$-dimensional semantic space. Our assumption is that an article would be indexed by its most related subject headings, i.e., the MeSH headings with the highest cosine similarities to the document itself. To evaluate this, we computed embeddings for term and MeSH headings using the training dataset (previously selected $10^{6}$ MEDLINE articles). We then prepared a separate testing dataset which contains $10^{4}$ articles randomly selected from WorldCat.org. The articles in the testing dataset all have an abstract and are indexed by at least one MeSH heading. For each of these articles, we computed the document embedding using the terms in its title and abstract, following Eq. 3 . We then computed their most similar MeSH headings and compared them with the actual ones. Notice how this method is, therefore, not biased towards predicting the more common (and often less informative) subjects.

For FastText and Sent2Vec, we did the same, i.e., using the document-subject similarities to select the potential candidates. Since FastText and Sent2Vec can be used to train a supervised text classifier (Joulin et al. 2017), we additionally trained a classifier where each article's title and abstract were concatenated as a text, and their actual MeSH subject headings were used as the labels to predict. We trained a separate FastText and Sent2Vec text classifier, which we used to predict the most likely subjects for the documents in the testing dataset, based on their title and abstract. The parameters for training a classifier were exactly the same as those for generating word embeddings, but the train time was dramatically shorter, less than 5 minutes with the same machine.

All candidate subjects were ranked, by their similarities to the document or by the probabilities according to the corresponding classifiers, as appropriate. Figure 1 shows the precision@ $n$ and recall@n for different methods/settings. Both FastText and Sent2Vec perform much worse than our method if using document-subject similarities for subject prediction. As multi-label classifiers, their performance are nearly identical to each other and the quality of the predicted subjects are comparable with our similarity-based prediction. Their precision@ $\mathrm{n}$ is higher than our method for low values of $n$ while it 

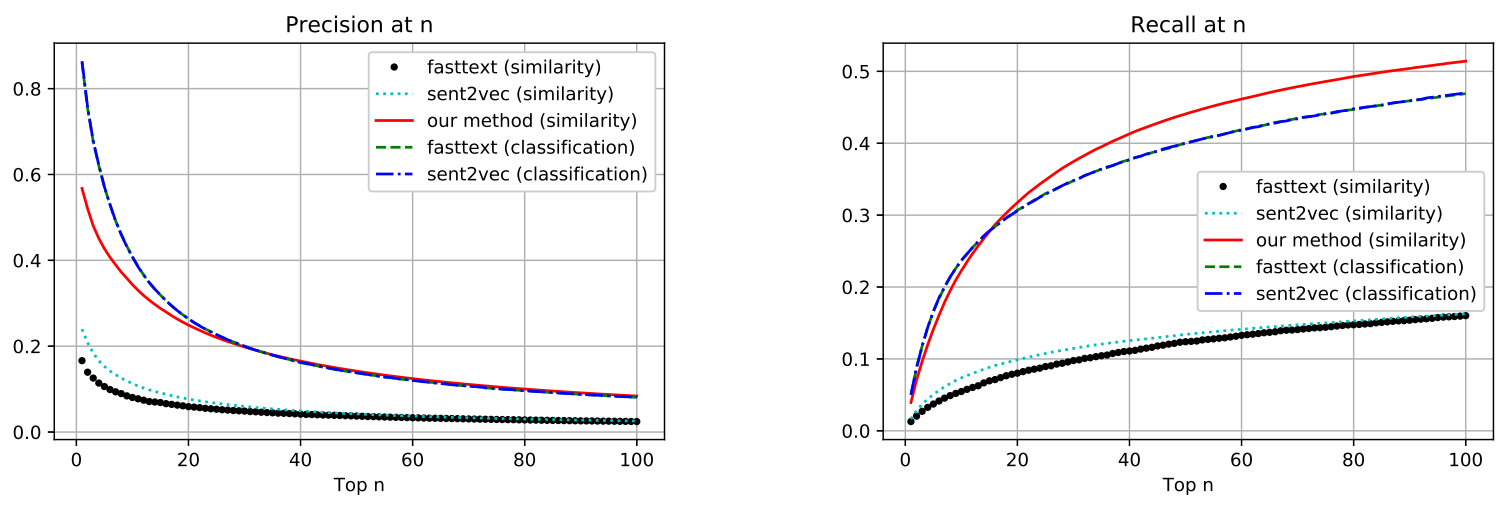

Figure 1: The performance comparison when predicting subjects

quickly decreases to be almost the same as ours. Up to top 20 candidates, the recall for three methods are more or less the same, but our method is able to predict more actual subjects at lower ranks, where the recall outperforms FastText and Sent2Vec.

Table 4 lists the 23 actual MeSH headings of an example article ${ }^{8}$ The MeSH terms that reflect the major points of this article are marked with an asterisk (*). The 25 most relevant MeSH headings predicted by three methods are also listed. It is not surprising that subjects such as "Humans" and "Female" are predicted first by FastText and Sent2Vec, because they are the most frequent ones used in the training dataset. In fact, many of the subjects predicted by these two classifiers are very common (see their document counts in Table 4). These classifiers have trouble finding subjects which describe the articles more precisely, while our method ranks specific subjects such as "Lens Capsule, Crystalline/Surgery" high in the list, even though fewer than 100 articles in the training set are indexed by this subject.

We realise that this evaluation has its limitations. As shown in Table 4 , highly related MeSH headings such as "Lenses Intraocular" and "Phacoemulsification Methods" are predicted as good candidates for this article, both of which are reasonable and potentially useful. But since they are not the subject headings that the professional taxonomists have chosen, their value cannot be easily assessed. This illustrates how precision/recall may not be a very meaningful evaluation metric in this application. It also shows how this method could provide good recommendations to cataloguers.

\section{Conclusion}

We have described a novel, simple, effective and efficient method for term and document embeddings. As we have shown, our method has important practical benefits: 1) it is fast and has low hardware requirements, having linear time complexity and constant space complexity in function of the number of documents, resulting in very short run-times in practice. 2) Since no iterative optimisation is needed, the resulting embeddings are not affected by parameter initialisation and there is no uncertainty about the quality of the results of a run. 3) It computes semantically discriminative term embeddings and weightings with a single pass through the training data, and has the capacity to effectively include very rare words. Our experiments show it outperforms state-of-the-art methods in terms of the STS benchmark and subject prediction when trained on the same datasets, while at the same time being computationally cheaper by orders of magnitude.

In the future, we will integrate sub-word information into the embedding process and evaluate how effectively previously unseen words can be embedded. We will consider a wider variety of evaluation methods, especially getting domain experts involved.

$\sqrt[8]{\text { https://www.ncbi.nlm.nih.gov/pubmed/14670424 }}$ 







\section{References}

Achlioptas, D. (2003). Database-friendly random projections: Johnson-Lindenstrauss with binary coins. Journal of Computer and System Sciences 66(4), 671-687.

Agirre, E., C. Banea, D. Cer, M. Diab, A. Gonzalez-Agirre, R. Mihalcea, G. Rigau, and J. Wieb (2016). Semeval-2016 task 1: Semantic textual similarity, monolingual and cross-lingual evaluation. In Proceedings of the SemEval-2016.

Arora, S., Y. Liang, and T. Ma (2017). A simple but tough-to-beat baseline for sentence embeddings. In Proceedings of 6th International Conference on Learning Representations. Poster.

Baroni, M., G. Dinu, and G. Kruszewski (2014). Don't count, predict ! A systematic comparison of context-counting vs . context-predicting semantic vectors. Proceedings of the 52nd Annual Meeting of the Association for Computational Linguistics., 238-247.

Bhatia, K., H. Jain, P. Kar, M. Varma, and P. Jain (2015). Sparse local embeddings for extreme multilabel classification. In C. Cortes, N. D. Lawrence, D. D. Lee, M. Sugiyama, and R. Garnett (Eds.), Advances in Neural Information Processing Systems 28, pp. 730-738. Curran Associates, Inc.

Bojanowski, P., E. Grave, A. Joulin, and T. Mikolov (2016). Enriching word vectors with subword information. arXiv preprint arXiv:1607.04606.

Cer, D., M. Diab, E. Agirre, I. Lopez-Gazpio, and L. Specia (2017). Semeval-2017 task 1: Semantic textual similarity multilingual and crosslingual focused evaluation. In Proceedings of the 11th International Workshop on Semantic Evaluation (SemEval-2017), Vancouver, Canada, pp. 1-14.

Conneau, A., D. Kiela, H. Schwenk, L. Barrault, and A. Bordes (2017). Supervised learning of universal sentence representations from natural language inference data. In Proceedings of the 2017 Conference on Empirical Methods in Natural Language Processing, pp. 670-680. Association for Computational Linguistics.

Dumais, S. T. (2005). Latent semantic analysis. Annual Review of Information Science and Technology $38,188-230$.

Foster, D. V. and P. Grassberger (2011, Jan). Lower bounds on mutual information. Phys. Rev. E 83, 010101.

Furnas, G. W., T. K. Landauer, L. M. Gomez, and S. T. Dumais (1983). Statistical semantics: Analysis of the potential performance of keyword information systems. Bell System Technical Journal 62(6), 17531806.

Harris, Z. (1954). Distributional structure. Word 10(23), 146162.

Johnson, W. and J. Lindenstrauss (1984). Extensions of Lipschitz mappings into a Hilbert space. Contemporary Math. 26, 189-206.

Joulin, A., E. Grave, P. Bojanowski, and T. Mikolov (2017). Bag of tricks for efficient text classification. In Proceedings of the 15th Conference of the European Chapter of the Association for Computational Linguistics: Volume 2, Short Papers, pp. 427-431. Association for Computational Linguistics.

Le, Q. V. and T. Mikolov (2014, 5). Distributed Representations of Sentences and Documents. International Conference on Machine Learning - ICML 2014 32, 11881196.

Liu, J., W.-C. Chang, Y. Wu, and Y. Yang (2017). Deep learning for extreme multi-label text classification. In Proceedings of the 40th International ACM SIGIR Conference on Research and Development in Information Retrieval, SIGIR '17, New York, NY, USA, pp. 115-124. ACM. 
Manning, C. D. and H. Schütze (1999). Foundations of Statistical Natural Language Processing. Cambridge, MA, USA: MIT Press.

Mikolov, T., I. Sutskever, K. Chen, G. Corrado, and J. Dean (2013). Distributed representations of words and phrases and their compositionality. In Proceedings of the 26th International Conference on Neural Information Processing Systems - Volume 2, NIPS'13, USA, pp. 3111-3119. Curran Associates Inc.

Moody, C. E. (2016, May). Mixing Dirichlet Topic Models and Word Embeddings to Make lda2vec. arXiv e-prints, arXiv:1605.02019.

Pagliardini, M., P. Gupta, and M. Jaggi (2018). Unsupervised learning of sentence embeddings using compositional n-gram features. In Proceedings of the 2018 Conference of the North American Chapter of the Association for Computational Linguistics: Human Language Technologies, Volume 1 (Long Papers), pp. 528-540. Association for Computational Linguistics.

Pearson, K. (1901). Liii. on lines and planes of closest fit to systems of points in space. The London, Edinburgh, and Dublin Philosophical Magazine and Journal of Science 2(11), 559-572.

Pennington, J., R. Socher, and C. D. Manning (2014). GloVe: Global vectors for word representation. In Empirical Methods in Natural Language Processing (EMNLP), pp. 1532-1543.

Peters, M. E., M. Neumann, M. Iyyer, M. Gardner, C. Clark, K. Lee, and L. Zettlemoyer (2018). Deep contextualized word representations. In Proceedings of the 16th Annual Conference of the North American Chapter of the Association for Computational Linguistics: Human Language Technologies.

Prabhu, Y. and M. Varma (2014). Fastxml: A fast, accurate and stable tree-classifier for extreme multilabel learning. In Proceedings of the 20th ACM SIGKDD International Conference on Knowledge Discovery and Data Mining, KDD '14, New York, NY, USA, pp. 263-272. ACM.

QasemiZadeh, B., L. Kallmeyer, and A. Herbelot (2017). Non-negative randomized word embeddings. In Proceedings of Traitement automatique des langues naturelles (TALN2017).

Roweis, S. T. and L. K. Saul (2000). Nonlinear dimensionality reduction by locally linear embedding. science 290(5500), 2323-2326.

Sahlgren, M. (2008). The distributional hypothesis. Rivista di Linguistica 20(1), 3353.

Trefethen, L. N. and D. Bau III (1997). Numerical linear algebra. Philadelphia: Society for Industrial and Applied Mathematics.

Weaver, W. (1955). Translation. In W. Locke and D. Booth (Eds.), Machine Translation of Languages, pp. 15-23. Cambridge, Massachusetts: MIT Press. 